\title{
Properties of nucleon in nuclear matter: once more
}

\author{
K. Azizi ${ }^{1, a}$, N. Er ${ }^{2, b}$ \\ ${ }^{1}$ Department of Physics, Doğuş University, Acıbadem-Kadıköy, 34722 Istanbul, Turkey \\ ${ }^{2}$ Department of Physics, Abant Izzet Baysal University, Gölköy Kampüsü, 14980 Bolu, Turkey
}

Received: 14 January 2014 / Accepted: 9 May 2014 / Published online: 27 May 2014

(C) The Author(s) 2014. This article is published with open access at Springerlink.com

\begin{abstract}
We calculate the mass and residue of the nucleon in nuclear matter in the frame work of QCD sum rules using the nucleon's interpolating current with an arbitrary mixing parameter. We evaluate the effects of the nuclear medium on these quantities and compare the results obtained with the existing theoretical predictions. The results are also compared with those obtained in vacuum to find the shifts in the quantities under consideration. Our calculations show that these shifts in the mass and residue are about 32 and $15 \%$, respectively.
\end{abstract}

\section{Introduction}

To analyze the experimental results on the relativistic heavy ion collision obtained at different experiments such as CERN, the European Organization for Nuclear Research, and BNL, Brookhaven National Laboratory, as well as for better understanding the internal structure of neutron stars, the in-medium properties of hadrons especially the properties of nucleons at nuclear medium are needed. On the experimental side, there has been progress on the in-medium properties of hadrons in recent years. The Facility for Antiproton and Ion Research (FAIR) and the Compressed Baryonic Matter (CBM) Collaboration at GSI intend to study the in-medium effects on the parameters of different hadrons. The Panda Collaboration, on the other hand, aims to concentrate on the properties of the charmed hadrons and study probable shifts on their masses and widths in nuclear medium $[1,2]$.

On the theoretical side, dozens of works have been devoted to the study of the nuclear matter and properties of hadrons, especially nucleons at dense medium. In [3], the basic properties of the nuclear matter are determined in the frame work of QCD sum rules as one of the most easily applicable and attractive tools to hadron physics. This method, then, has been applied to some finite-density problems [4-7]. In [4,5],

\footnotetext{
a e-mail: kazizi@dogus.edu.tr

be-mail: nuray@ibu.edu.tr
}

the authors have used the finite-density sum rules to investigate the saturation properties of nuclear matter. In series of papers [8-10], T. D. Cohen et al., have applied the QCD sum rules to relativistic nuclear physics and studied the effects of nuclear matter on the mass of the nucleons mostly for the Ioffe current. Only in [10], the authors extended the Ioffe current ( $\beta=-1$ ) with $\beta$ being the mixing parameter in the interpolating current of the nucleons to $-1.15 \leq \beta \leq-0.95$ using the mass sum rules. For some studies of the nucleon mass shift in the nuclear medium for the Ioffe current and properties of other hadrons in a dense medium, see for instance [11-28]. The effects of four-quark condensate on the nucleon parameters have also been studied in [29]. Recently, the QCD sum rules have been used to analyze the residue of the nucleon pole as a function of the nuclear density [30] using a special current corresponding to an axial-vector diquark coupled to a quark. Note that the mass and residue of nucleon have also been investigated in an instanton medium very recently in [31].

In this article, we extend the previous studies to calculate both the mass and residue of the nucleon in nuclear matter using the interpolating current with an arbitrary mixing parameter in the frame work of QCD sum rules. As the mass sum rule is the ratio of two sum rules (according to the method used), it may not lead to a reliable region for the arbitrary mixing parameter. The unstable points of two sum rules in nominator and denominator generally coincide and cancel each other and the mass shows roughly good stability with respect to the mixing parameter in the whole interval $(-\infty,+\infty)$ for $\beta$. Hence, to restrict this parameter, the only reliable chance is to use the sum rule for the residue as it does not contain any ratio of sum rules and includes only one function from the operator product expansion (OPE) side. In this connection, we use the sum rule for the residue to find the working region for the mixing parameter $\beta$. In this way, we extend the previous calculations [30] on the residue as our working region for $\beta$ includes the Ioffe current used in [30] to discuss the behavior of the residue of the nucleon with 
respect to the nuclear density. We also extend the study [10] on the mass of the nucleon in nuclear matter by the extension of the working region for the mixing parameter. Using the working region obtained for $\beta$ as well as working regions of other auxiliary parameters entering the sum rules, we then obtain the shifts in the values of mass and residue compared to their vacuum values. We also compare our results on the mass and residue of the nucleon with the existing numerical results obtained via the Ioffe current in vacuum. Finally, we extract the vector and scalar self-energies of the nucleon in nuclear matter and compare the obtained results with the predictions of model independent studies [32,33].

Our results on the mass and residue can be used in theoretical calculations via the current under consideration such as the computation of the electromagnetic properties and multiple moments of the nucleon and the strong coupling constants of the nucleon to other hadrons in nuclear medium. The study of the electromagnetic properties of nucleons has been in focus of many experimental and theoretical works for many years. Unfortunately, there is no a good consistency among the results obtained in different ways on the electromagnetic form factors (see for instance [34] and references therein). In [34] it is shown that the Ioffe current fails to reproduce some experimental data on some of electromagnetic form factors of the nucleon in vacuum. Also, many previous works on the strong coupling constants among various groups of baryons including the nucleons with different mesonic groups (see for instance $[35,36])$ reveal that the Ioffe current remains out of the reliable region and some other values of the mixing parameter $\beta$ for octet baryons are favored. We guess that the problem of the Ioffe current failing to explain some electromagnetic and strong coupling parameters in vacuum will still occur also in the nuclear medium. When we calculate the electromagnetic or strong parameters of nucleon in nuclear matter using the current with an arbitrary mixing parameter, we immediately need their masses and residues calculated via the same current in nuclear matter. Our working region for the parameter $\beta$ as well as our predictions on the mass and residue of the nucleon can be useful in this respect.

The article is organized as follows: In Sect. 2, we obtain QCD sum rules for the mass and residue of the nucleon in the nuclear matter. Section 3 is devoted to numerical analyses of the sum rules and our comparison of the results with the existing predictions. Section 4 contains our concluding remarks.

\section{QCD sum rules for the mass and residue of nucleon in nuclear matter}

To obtain the sum rules for the mass and residue of nucleon in nuclear matter, the starting point is to consider the following two-point correlationfunction:
$\Pi(p)=i \int \mathrm{d}^{4} x e^{i p \cdot x}\left\langle\psi_{0}|T[J(x) \bar{J}(0)]| \psi_{0}\right\rangle$,

where $p$ is the four momentum of the nucleon and $\left|\psi_{0}\right\rangle$ is the nuclear matter ground state. The nucleon interpolating current is taken as

$J(x)=2 \epsilon_{a b c} \Sigma_{i=1}^{2}\left[q_{1}^{T, a}(x) C A_{1}^{i} q_{2}^{b}(x)\right] A_{2}^{i} q_{1}^{c}(x)$,

where $a, b, c$ are color indices, $C$ is the charge conjugation operator, and $A_{1}^{1}=I, A_{1}^{2}=A_{2}^{1}=\gamma_{5}, A_{2}^{2}=\beta$. As previously said, the parameter $\beta$ is an arbitrary auxiliary parameter, and $\beta=-1$ corresponds to the Ioffe current (for some discussions as regards the nucleon interpolating currents see for instance $[29,31,37,38])$. The quark flavors for the proton (neutron) are $q_{1}=u$ and $q_{2}=d\left(q_{1}=d\right.$ and $\left.q_{2}=u\right)$. Here we will adopt the isospin symmetry, thus treating the proton and neutron as a nucleon.

From the general philosophy of the method under consideration, we calculate the above mentioned correlation function from two different windows: in terms of hadronic parameters called the phenomenological or hadronic side and in terms of QCD degrees of freedom using the OPE at the nuclear medium named OPE or theoretical side. Equating these two sides, we obtain QCD sum rules for the mass and residue in nuclear matter. To suppress the contribution of the higher states and continuum, a Borel transformation and continuum subtraction $[39,40]$ are applied to both sides of the sum rules obtained.

\subsection{Hadronic side}

On the hadronic side, the correlation function is calculated inserting a complete set of nucleon states with the same quantum numbers as the interpolating current. After performing the integral over four- $x$, we get

$$
\Pi^{\mathrm{Had}}(p)=-\frac{\left\langle\psi_{0}|J(x)| N(p, s)\right\rangle\left\langle N(p, s)|\bar{J}(0)| \psi_{0}\right\rangle}{p^{2}-m_{N}^{* 2}}+\ldots,
$$

where the dots represent the contributions of higher states and the continuum and $m_{N}^{*}$ is the modified mass of the nucleon in nuclear matter. The matrix element of the interpolating current between the nucleon ground state and the baryonic state is parametrized as

$\left\langle\psi_{0}|J(x)| N(p, s)\right\rangle=\lambda_{N}^{*} u(p, s)$,

where $\lambda_{N}^{*}$ is the modified residue or the coupling strength of the nucleon current $J(x)$ to the nucleon quasi-particle in the nuclear matter and $u(p, s)$ is the positive energy Dirac spinor. Using Eq. (4) in Eq. (3), we get

$$
\Pi^{\mathrm{Had}}(p)=-\frac{\lambda_{N}^{* 2}\left(\not p+m_{N}^{*}\right)}{p^{2}-m_{N}^{* 2}}+\cdots=-\frac{\lambda_{N}^{* 2}}{\left(\not p-m_{N}^{*}\right)}+\ldots
$$


Considering the interactions between the nucleon and the nuclear matter, the hadronic side of the correlation function takes the modified form

$\Pi^{\mathrm{Had}}(p)=-\frac{\lambda_{N}^{* 2}}{\left(p^{\mu}-\Sigma_{v}^{\mu}\right) \gamma_{\mu}-\left(m_{N}+\Sigma_{S}\right)}+\ldots$,

where $\Sigma_{\nu}^{\mu}$ and $\Sigma_{S}$ are the vector and scalar self-energies of the nucleon in nuclear matter, respectively [11]. In general, we can write

$\Sigma_{v}^{\mu}=\Sigma_{\nu} u^{\mu}+\Sigma_{v}^{\prime} p^{\mu}$,

where $\Sigma_{v}$ and $\Sigma_{v}^{\prime}$ are constants and $u^{\mu}$ is the four-velocity of the nuclear medium. Here we neglect $\Sigma_{\nu}^{\prime}$ due to its small contribution (see also [11]). Apart from the vacuum QCD calculations, the four-velocity of the nuclear matter is a new concept that causes extra structures for the correlation function. We shall work in the rest frame of the nucleon with $u^{\mu}=(1,0)$. By substitution of Eq. (7) into Eq. (6), the hadronic side of the correlation function becomes

$\Pi^{\mathrm{Had}}(p)=-\frac{\lambda_{N}^{* 2}}{\left(\not p-\Sigma_{v} \not \ell\right)-\left(m_{N}+\Sigma_{S}\right)}+\ldots$,

which can be written in terms of the three different structures as

$$
\begin{aligned}
\Pi^{\mathrm{Had}}(p)= & \Pi_{p}^{\mathrm{Had}}\left(p^{2}, p_{0}\right) \not p+\Pi_{u}^{\mathrm{Had}}\left(p^{2}, p_{0}\right) \not \iota \\
& +\Pi_{S}^{\mathrm{Had}}\left(p^{2}, p_{0}\right) I,
\end{aligned}
$$

where $p_{0}$ is the energy of the quasi-particle, $I$ is the unit matrix, and

$$
\begin{aligned}
& \Pi_{p}^{\mathrm{Had}}\left(p^{2}, p_{0}\right)=-\lambda_{N}^{* 2} \frac{1}{p^{2}-\mu^{2}}, \\
& \Pi_{u}^{\mathrm{Had}}\left(p^{2}, p_{0}\right)=+\lambda_{N}^{* 2} \frac{\Sigma_{v}}{p^{2}-\mu^{2}}, \\
& \Pi_{S}^{\mathrm{Had}}\left(p^{2}, p_{0}\right)=-\lambda_{N}^{* 2} \frac{m_{N}^{*}}{p^{2}-\mu^{2}} .
\end{aligned}
$$

Here $m_{N}^{*}=m_{N}+\Sigma_{S}$ and $\mu^{2}=m_{N}^{* 2}-\Sigma_{v}^{2}+2 p_{0} \Sigma_{v}$. After a Wick rotation and applying the Borel transformation with respect to $p^{2}$, we get

$$
\begin{aligned}
& \hat{B} \Pi_{p}^{\mathrm{Had}}\left(p^{2}, p_{0}\right)=-\lambda_{N}^{* 2} e^{-\mu^{2} / M^{2}}, \\
& \hat{B} \Pi_{u}^{\mathrm{Had}}\left(p^{2}, p_{0}\right)=+\lambda_{N}^{* 2} \Sigma_{\nu} e^{-\mu^{2} / M^{2}}, \\
& \hat{B} \Pi_{S}^{\mathrm{Had}}\left(p^{2}, p_{0}\right)=-\lambda_{N}^{* 2} m_{N}^{*} e^{-\mu^{2} / M^{2}} .
\end{aligned}
$$

\subsection{OPE side}

The OPE side of the correlation function can be calculated in the deep Euclidean region. This function can also be written in terms of different structures as

$\Pi^{\mathrm{OPE}}(p)=\Pi_{p}^{\mathrm{OPE}} \not p+\Pi_{u}^{\mathrm{OPE}} \not \iota+\Pi_{S}^{\mathrm{OPE}} I$.
Each $\Pi_{i}^{\mathrm{OPE}}$ function, where $i=\not p, \mu$, and where $I$ can be written in terms of a dispersion integral, is

$\Pi_{i}^{\mathrm{OPE}}=\int \frac{\rho_{i}(s)}{s-p^{2}} \mathrm{~d} s$,

where $\rho_{i}(s)=\frac{1}{\pi} \operatorname{Im}\left[\Pi_{i}^{\mathrm{OPE}}\right]$ are the spectral densities. Using the explicit form of the interpolating current in the correlation function of Eq. (1) and contracting out all quark pairs via Wick's theorem, we find

$$
\begin{aligned}
\Pi^{\mathrm{OPE}}(p)= & -4 i \epsilon_{a b c} \epsilon_{a^{\prime} b^{\prime} c^{\prime}} \int \mathrm{d}^{4} x e^{i p x}\left\langle\psi_{0}\right| \\
& \times\left\{\left(\gamma_{5} S_{u}^{c b^{\prime}}(x) S_{d}^{\prime b a^{\prime}}(x) S_{u}^{a c^{\prime}}(x) \gamma_{5} .\right.\right. \\
& \left.-\gamma_{5} S_{u}^{c c^{\prime}}(x) \gamma_{5} \operatorname{Tr}\left[S_{u}^{a b^{\prime}}(x) S_{d}^{\prime b a^{\prime}}(x)\right]\right) \\
& +\beta\left(\gamma_{5} S_{u}^{c b^{\prime}}(x) \gamma_{5} S_{d}^{\prime b a^{\prime}}(x) S_{u}^{a c^{\prime}}(x)\right. \\
& +S_{u}^{c b^{\prime}}(x) S_{d}^{\prime b a^{\prime}}(x) \gamma_{5} S_{u}^{a c^{\prime}}(x) \gamma_{5} \\
& -\gamma_{5} S_{u}^{c c^{\prime}}(x) \operatorname{Tr}\left[S_{u}^{a b^{\prime}}(x) \gamma_{5} S_{d}^{\prime b a^{\prime}}(x)\right] \\
& \left.-S_{u}^{c c^{\prime}}(x) \gamma_{5} \operatorname{Tr}\left[S_{u}^{a b^{\prime}}(x) S_{d}^{\prime b a^{\prime}}(x) \gamma_{5}\right]\right) \\
& +\beta^{2}\left(S_{u}^{c b^{\prime}}(x) \gamma_{5} S_{d}^{\prime b a^{\prime}}(x) \gamma_{5} S_{u}^{a c^{\prime}}(x)\right. \\
& \left.\left.-S_{u}^{c c^{\prime}}(x) \operatorname{Tr}\left[S_{d}^{b a^{\prime}}(x) \gamma_{5} S_{u}^{\prime a b^{\prime}}(x) \gamma_{5}\right]\right)\right\}\left|\psi_{0}\right\rangle
\end{aligned}
$$

where $S^{\prime}=C S^{T} C, S_{u, d}$ are light-quark propagators and $\operatorname{Tr}[\ldots]$ denotes the trace of the gamma matrices. In coordinate-space, the light-quark propagator at the nuclear medium has the following form in the fixed-point gauge $[15,41]$ :

$$
\begin{aligned}
S_{q}^{a b}(x) \equiv & \left\langle\psi_{0}\left|T\left[q^{a}(x) \bar{q}^{b}(0)\right]\right| \psi_{0}\right\rangle_{\rho_{N}} \\
= & \frac{i}{2 \pi^{2}} \delta^{a b} \frac{1}{\left(x^{2}\right)^{2}} \not x-\frac{m_{q}}{4 \pi^{2}} \delta^{a b} \frac{1}{x^{2}} \\
& +\chi_{q}^{a}(x) \bar{\chi}_{q}^{b}(0)-\frac{i g_{s}}{32 \pi^{2}} F_{\mu \nu}^{A}(0) t^{a b, A} \\
& \times \frac{1}{x^{2}}\left[\not x \sigma^{\mu \nu}+\sigma^{\mu \nu} \not x\right]+\ldots,
\end{aligned}
$$

where $\rho_{N}$ is the nuclear matter density, $\chi_{q}^{a}$ and $\bar{\chi}_{q}^{b}$ are the Grassmann background quark fields, $F_{\mu \nu}^{A}$ is the classical background gluon field, and the first and second terms are the expansion of the free quark propagator to first order in the quark mass (perturbative part); the third and fourth terms are the contributions due to the background quark and gluon fields (non-perturbative part), respectively. The gluonic contribution to the above equation corresponds to a single gluon interaction keeping only the leading term in the short-distance expansion of the gluon field. We ignore contributions of the derivatives of the gluon field tensor as well as additional gluon interactions in the expression of the lightquark propagator (see also [15]). When using Eq. (15) in Eq. (14), we will end up with the products of the Grassmann background quark fields and classical background gluon fields which correspond to the ground-state matrix elements of the corresponding quark and gluon operators [15], 
$\chi_{a \alpha}^{q}(x) \bar{\chi}_{b \beta}^{q}(0)=\left\langle q_{a \alpha}(x) \bar{q}_{b \beta}(0)\right\rangle_{\rho_{N}}$,

$F_{\kappa \lambda}^{A} F_{\mu \nu}^{B}=\left\langle G_{\kappa \lambda}^{A} G_{\mu \nu}^{B}\right\rangle_{\rho_{N}}$,

$\chi_{a \alpha}^{q} \bar{\chi}_{b \beta}^{q} F_{\mu \nu}^{A}=\left\langle q_{a \alpha} \bar{q}_{b \beta} G_{\mu \nu}^{A}\right\rangle_{\rho_{N}}$,

$\chi_{a \alpha}^{q} \bar{\chi}_{b \beta}^{q} \chi_{c \gamma}^{q} \bar{\chi}_{d \delta}^{q}=\left\langle q_{a \alpha} \bar{q}_{b \beta} q_{c \gamma} \bar{q}_{d \delta}\right\rangle_{\rho_{N}}$.

To proceed, we need to define the quark and gluon and mixed condensates in nuclear matter. The matrix element $\left\langle q_{a \alpha}(x) \bar{q}_{b \beta}(0)\right\rangle_{\rho_{N}}$ is projected out as [15]

$$
\begin{aligned}
\left\langle q_{a \alpha}(x) \bar{q}_{b \beta}(0)\right\rangle_{\rho_{N}}= & -\frac{\delta_{a b}}{12}\left[\left(\langle\bar{q} q\rangle_{\rho_{N}}+x^{\mu}\left\langle\bar{q} D_{\mu} q\right\rangle_{\rho_{N}}\right.\right. \\
& \left.+\frac{1}{2} x^{\mu} x^{\nu}\left\langle\bar{q} D_{\mu} D_{\nu} q\right\rangle_{\rho_{N}}+\ldots\right) \delta_{\alpha \beta} \\
& +\left(\left\langle\bar{q} \gamma_{\lambda} q\right\rangle_{\rho_{N}}+x^{\mu}\left\langle\bar{q} \gamma_{\lambda} D_{\mu} q\right\rangle_{\rho_{N}}\right. \\
& \left.\left.+\frac{1}{2} x^{\mu} x^{\nu}\left\langle\bar{q} \gamma_{\lambda} D_{\mu} D_{\nu} q\right\rangle_{\rho_{N}}+\ldots\right) \gamma_{\alpha \beta}^{\lambda}\right] .
\end{aligned}
$$

The quark-gluon condensate in nuclear matter is written as

$$
\begin{aligned}
\left\langle g_{s} q_{a \alpha} \bar{q}_{b \beta} G_{\mu \nu}^{A}\right\rangle_{\rho_{N}}= & -\frac{t_{a b}^{A}}{96}\left\{\left\langle g_{s} \bar{q} \sigma \cdot G q\right\rangle_{\rho_{N}}\right. \\
& \times\left[\sigma_{\mu \nu}+i\left(u_{\mu} \gamma_{\nu}-u_{\nu} \gamma_{\mu}\right) \not u\right]_{\alpha \beta} \\
& +\left\langle g_{s} \bar{q} \not h \sigma \cdot G q\right\rangle_{\rho_{N}} \\
& \times\left[\sigma_{\mu \nu} \not h+i\left(u_{\mu} \gamma_{\nu}-u_{\nu} \gamma_{\mu}\right)\right]_{\alpha \beta} \\
& -4\left(\langle\bar{q} u \cdot D u \cdot D q\rangle_{\rho_{N}}\right. \\
& \left.+i m_{q}\langle\bar{q} \not h u \cdot D q\rangle_{\rho_{N}}\right) \\
& \left.\times\left[\sigma_{\mu \nu}+2 i\left(u_{\mu} \gamma_{\nu}-u_{\nu} \gamma_{\mu}\right) \not l\right]_{\alpha \beta}\right\},
\end{aligned}
$$

where $t_{a b}^{A}$ are the Gell-Mann matrices and $D_{\mu}=\frac{1}{2}\left(\gamma_{\mu} D+\right.$ $\left.\not D \gamma_{\mu}\right)$. The matrix element of the four-dimension gluon condensate can also be written as

$$
\begin{aligned}
\left\langle G_{\kappa \lambda}^{A} G_{\mu \nu}^{B}\right\rangle_{\rho_{N}}= & \frac{\delta^{A B}}{96}\left[\left\langle G^{2}\right\rangle_{\rho_{N}}\left(g_{\kappa \mu} g_{\lambda \nu}-g_{\kappa \nu} g_{\lambda \mu}\right)\right. \\
& \left.+O\left(\left\langle\mathbf{E}^{2}+\mathbf{B}^{2}\right\rangle_{\rho_{N}}\right)\right],
\end{aligned}
$$

where we neglect the last term in this equation because of its small contribution. The various condensates in the above equations are defined as $[9,15]$

$$
\begin{aligned}
\left\langle\bar{q} \gamma_{\mu} q\right\rangle_{\rho_{N}}= & \langle\bar{q} \not h q\rangle_{\rho_{N}} u_{\mu}, \\
\left\langle\bar{q} D_{\mu} q\right\rangle_{\rho_{N}}=\langle\bar{q} u \cdot D q\rangle_{\rho_{N}} u_{\mu}=-i m_{q}\langle\bar{q} \not h q\rangle_{\rho_{N}} u_{\mu}, & \\
\left\langle\bar{q} \gamma_{\mu} D_{\nu} q\right\rangle_{\rho_{N}}= & \frac{4}{3}\langle\bar{q} \not u u \cdot D q\rangle_{\rho_{N}}\left(u_{\mu} u_{\nu}-\frac{1}{4} g_{\mu \nu}\right) \\
& +\frac{i}{3} m_{q}\langle\bar{q} q\rangle_{\rho_{N}}\left(u_{\mu} u_{\nu}-g_{\mu \nu}\right)
\end{aligned}
$$

$$
\begin{aligned}
& \left\langle\bar{q} D_{\mu} D_{\nu} q\right\rangle_{\rho_{N}}=\frac{4}{3}\langle\bar{q} u \cdot D u \cdot D q\rangle_{\rho_{N}}\left(u_{\mu} u_{v}-\frac{1}{4} g_{\mu \nu}\right) \\
& -\frac{1}{6}\left\langle g_{s} \bar{q} \sigma \cdot G q\right\rangle_{\rho_{N}}\left(u_{\mu} u_{v}-g_{\mu \nu}\right),
\end{aligned}
$$

$$
\begin{aligned}
& \left\langle\bar{q} \gamma_{\lambda} D_{\mu} D_{\nu} q\right\rangle_{\rho_{N}}=2\langle\bar{q} \not u u \cdot D u \cdot D q\rangle_{\rho_{N}} \\
& \quad \times\left[u_{\lambda} u_{\mu} u_{\nu}-\frac{1}{6}\left(u_{\lambda} g_{\mu \nu}+u_{\mu} g_{\lambda \nu}+u_{\nu} g_{\lambda \mu}\right)\right] \\
& \quad-\frac{1}{6}\left\langle g_{s} \bar{q} \not h \sigma \cdot G q\right\rangle_{\rho_{N}}\left(u_{\lambda} u_{\mu} u_{\nu}-u_{\lambda} g_{\mu \nu}\right),
\end{aligned}
$$

where the equations of motion have been used and $O\left(m_{q}^{2}\right)$ terms have been neglected due to their very small contributions [15]. Now, we use the expressions of the light-quark propagator in the nuclear medium and different condensates presented above in Eq. (14) and perform the four-integral over $x$ to go to the momentum space. To suppress the contributions of the higher states and continuum we apply the Borel transformation with respect to the momentum squared and perform a continuum subtraction. We also use the quarkhadron duality assumption. The $\Pi_{i}^{\mathrm{OPE}}$ functions can be written in terms of the even and odd parts in terms of $p_{0}$ as

$\Pi_{i}^{\mathrm{OPE}}=\Pi_{i}^{E}+p_{0} \Pi_{i}^{O}$

where, after lengthy calculations, for the invariant functions $\Pi_{i}^{E, O}$ in the Borel scheme we get

$$
\begin{aligned}
\hat{B} \Pi_{p}^{E}= & -\frac{1}{256 \pi^{4}} \int_{0}^{s_{0}} \mathrm{~d} s e^{-s / M^{2}} s^{2}[5+\beta(2+5 \beta)] \\
& +\frac{1}{72 \pi^{2}} \int_{0}^{s_{0}} \mathrm{~d} s e^{-s / M^{2}}\left\{-8[5+\beta(2+5 \beta)] m_{q}\langle\bar{q} q\rangle_{\rho_{N}}\right. \\
& +9(-1+\beta)\left[3(1+\beta) m_{d}+2 m_{u}+4 \beta m_{u}\right]\langle\bar{q} q\rangle_{\rho_{N}} \\
& \left.+5[5+\beta(2+5 \beta)]\left\langle q^{\dagger} i D_{0} q\right\rangle_{\rho_{N}}\right\} \\
& -\frac{\left\langle g_{s}^{2} G^{2}\right\rangle_{\rho_{N}}}{1024 \pi^{4}} \mathrm{~d} s e^{-s / M^{2}}\left(6+\beta+5 \beta^{2}\right) \\
& +\frac{1}{192 M^{2} \pi^{2}}\{(-1+\beta) \\
& \times\left[-\left(40(1+\beta) m_{d}+(26+43 \beta) m_{u}\right) M^{2}\right. \\
& \left.\left.+8\left(3(1+\beta) m_{d}+2 m_{u}+4 \beta m_{u}\right) p_{0}^{2}\right]\right\}\left\langle\bar{q} g_{s} \sigma G q\right\rangle_{\rho_{N}} \\
& -\frac{1}{48 M^{2} \pi^{2}}\left\{( - 1 + \beta ) \left[(1+5 \beta) m_{u} M^{2}-32(1+2 \beta)\right.\right. \\
& \left.\left.\times m_{u} p_{0}^{2}-4(1+\beta) m_{d}\left(M^{2}+12 p_{0}^{2}\right)\right]\right\}\left\langle\bar{q} i D_{0} i D_{0} q\right\rangle_{\rho_{N}} \\
& -\frac{1}{144 \pi^{2}}\left\{\left[3(\beta-1) m_{q}\left(4(1+\beta) m_{d}-(1+5 \beta) m_{u}\right)\right.\right. \\
& \left.\left.+16(5+\beta(2+5 \beta)) p_{0}^{2}\right]\right\}\left\langle q^{\dagger} i D_{0} q\right\rangle_{\rho_{N}} \\
& \frac{1}{36 \pi^{2}}\left\{[5+\beta(2+5 \beta)] m_{q} p_{0}^{2}\right\}\langle\bar{q} q\rangle_{\rho_{N}},
\end{aligned}
$$




$$
\begin{aligned}
\hat{B} & \Pi_{p}^{O}=\frac{1}{72 \pi^{2}} \int_{0}^{s_{0}} \mathrm{~d} s e^{-s / M^{2}} \\
& \times\left\{15\left\langle q^{\dagger} q\right\rangle_{\rho_{N}}+3 \beta(2+5 \beta)\left\langle q^{\dagger} q\right\rangle_{\rho_{N}}\right\} \\
& +\frac{1}{576 M^{2} \pi^{2}}\left\{-3(1+3 \beta(2+\beta)) M^{2}\right. \\
& \left.+8(5+\beta(2+5 \beta)) p_{0}^{2}\right\}\left\langle q^{\dagger} g_{s} \sigma G q\right\rangle_{\rho_{N}} \\
& -\frac{1}{12 M^{2} \pi^{2}}\left\{[5+\beta(2+5 \beta)]\left(M^{2}-2 p_{0}^{2}\right)\right\}\left\langle q^{\dagger} i D_{0} i D_{0} q\right\rangle_{\rho_{N}} \\
& -\frac{1}{4 \pi^{2}}\left\{(\beta-1) m_{q}\left[3(1+\beta) m_{d}+(2+4 \beta) m_{u}\right]\right\}\left\langle q^{\dagger} q\right\rangle_{\rho_{N}},
\end{aligned}
$$

$$
\begin{aligned}
\hat{B} & \Pi_{u}^{E}(p)=\frac{1}{72 \pi^{2}} \int_{0}^{s_{0}} \mathrm{~d} s e^{-s / M^{2}}[-3(5+\beta(2+5 \beta)) \\
& \times\left\langle q^{\dagger} g_{s} \sigma G q\right\rangle_{\rho_{N}} \\
& -9(-1+\beta) m_{q}\left(3(1+\beta) m_{d}+2 m_{u}(1+2 \beta)\right)\left\langle q^{\dagger} q\right\rangle_{\rho_{N}} \\
& \left.\left.+3\left\langle q^{\dagger} q\right\rangle_{\rho_{N}} s\right)\right]+\frac{1}{128 \pi^{2}} \int_{0}^{s_{0}} \mathrm{~d} s e^{-s / M^{2}} \\
& \times 5\left(1+\beta^{2}\right)\left\langle q^{\dagger} g_{s} \sigma G q\right\rangle_{\rho_{N}} \\
& +\frac{1}{24 \pi^{2}}[5+\beta(2+5 \beta)] p_{0}^{2}\left\langle q^{\dagger} g_{s} \sigma G q\right\rangle_{\rho_{N}} \\
& +\frac{1}{2 \pi^{2}}[5+\beta(2+5 \beta)] p_{0}^{2}\left\langle q^{\dagger} i D_{0} i D_{0} q\right\rangle_{\rho_{N}},
\end{aligned}
$$

$\hat{B} \Pi_{u}^{O}(p)=\frac{1}{72 \pi^{2}} \int_{0}^{s_{0}} \mathrm{~d} s e^{-s / M^{2}}\left[5(5+\beta(2+5 \beta)) m_{q}\langle\bar{q} q\rangle_{\rho_{N}}\right.$

$$
+2(5+\beta(2+5 \beta))\left(-10\left\langle q^{\dagger} i D_{0} q\right\rangle_{\rho_{N}}\right.
$$$$
+\frac{1}{96 \pi^{2}}\left\{(\beta-1)\left[8(1+\beta) m_{d}+3(3+7 \beta) m_{u}\right]\right\}
$$$$
\times\left\langle\bar{q} g_{s} \sigma G q\right\rangle_{\rho_{N}}
$$$$
+\frac{1}{12 \pi^{2}}\left\{(\beta-1)\left[8(1+\beta) m_{d}+3(3+7 \beta) m_{u}\right]\right\}
$$$$
\times\left\langle\bar{q} i D_{0} i D_{0} q\right\rangle_{\rho_{N}}
$$$$
+\frac{1}{12 \pi^{2}}\left\{(\beta-1) m_{q}\left[4(1+\beta) m_{d}-(1+5 \beta) m_{u}\right]\right\}
$$$$
\times\left\langle q^{\dagger} i D_{0} q\right\rangle_{\rho_{N}},
$$

$$
\begin{aligned}
& \Pi_{S}^{E}(p)=-\frac{1}{64 \pi^{4}} \int_{0}^{s_{0}} \mathrm{~d} s e^{-s / M^{2}} s^{2}\left[(\beta-1)^{2} m_{d}+6\left(\beta^{2}-1\right) m_{u}\right] \\
& -\frac{1}{32 \pi^{2}}(\beta-1) \int_{0}^{s_{0}} \mathrm{~d} s e^{-s / M^{2}}\left\{\left(( 5 + 7 \beta ) \left\langle\bar{q} g_{s} \sigma\right.\right.\right. \\
& \left.\quad \times G q\rangle_{\rho_{N}}\right)+4 m_{q}\left[(\beta-1) m_{d}+6(\beta+1) m_{u}\right] \\
& \left.\quad \times\langle\bar{q} q\rangle_{\rho_{N}}-2(5+7 \beta) s\langle\bar{q} q\rangle_{\rho_{N}}\right\}
\end{aligned}
$$

$$
\begin{aligned}
& +\frac{\left\langle g_{s}^{2} G^{2}\right\rangle_{\rho_{N}}}{512 \pi^{4}}(\beta-1) \int_{0}^{s_{0}} \mathrm{~d} s e^{-s / M^{2}}\left[\beta m_{d}-6(1+\beta) m_{u}\right] \\
& +\frac{1}{128 \pi^{4}}(\beta-1) \beta \int_{0}^{s_{0}} \mathrm{~d} s e^{-s / M^{2}}\left\langle\bar{q} g_{s} \sigma G q\right\rangle_{\rho_{N}} \\
& +\frac{1}{192 \pi^{2}}(\beta-1)(20+29 \beta) p_{0}^{2}\left\langle\bar{q} g_{s} \sigma G q\right\rangle_{\rho_{N}} \\
& -\frac{1}{24 \pi^{2}}[20+(9-29 \beta) \beta] p_{0}^{2}\left\langle\bar{q} i D_{0} i D_{0} q\right\rangle_{\rho_{N}} \\
& +\frac{1}{12 \pi^{2}}(\beta-1) m_{q}\left[(\beta-1) m_{d}+6(\beta+1) m_{u}\right] p_{0}^{2}\langle\bar{q} q\rangle_{\rho_{N}},
\end{aligned}
$$

and

$$
\begin{aligned}
\Pi_{S}^{O}(p)= & -\frac{1}{32 \pi^{2}}(\beta-1) \int_{0}^{s_{0}} \mathrm{~d} s e^{-s / M^{2}} \\
& \times\left\{4 \left[m_{q}(5+7 \beta)+m_{d}(1-\beta)\right.\right. \\
& \left.\left.-6(1+\beta) m_{u}\right]\left\langle q^{\dagger} q\right\rangle_{\rho_{N}}\right\} \\
& +\frac{1}{192 M^{2} \pi^{2}}(\beta-1)\left\{3+(8+7 \beta) m_{u} M^{2}\right. \\
& +48(1+\beta) m_{u} p_{0}^{2} \\
& \left.+4 m_{d}\left[M^{2}(1-4 \beta)+2(\beta-1) p_{0}^{2}\right]\right\}\left\langle q^{\dagger} g_{s} \sigma G q\right\rangle_{\rho_{N}} \\
& +\frac{1}{4 M^{2} \pi^{2}}(\beta-1)\left[(\beta-1) m_{d}+6(\beta+1) m_{u}\right] \\
& \times\left(M^{2}+2 p_{0}^{2}\right)\left\langle q^{\dagger} i D_{0} i D_{0} q\right\rangle_{\rho_{N}} \\
& -\frac{1}{24 \pi^{2}}(\beta-1)\left[\beta m_{q}-8 m_{d}(1-\beta)\right. \\
& \left.+48(1+\beta) m_{u}\right]\left\langle q^{\dagger} i D_{0} q\right\rangle_{\rho_{N}},
\end{aligned}
$$

where $s_{0}$ is the continuum threshold. Having calculated both the hadronic and the OPE sides of the correlation function, now we equate these two sides for all structures to find the corresponding QCD sum rules. For instance, in the case of the structure $\not p$, we have

$$
-\lambda_{N}^{* 2} e^{-\mu^{2} / M^{2}}=\hat{B} \Pi_{p}^{\mathrm{OPE}}
$$

To find the mass sum rule, we eliminate $\lambda_{N}^{* 2}$ in the above equation, as a result of which we get

$\mu^{2}=\frac{\frac{\partial}{\partial\left(-\frac{1}{M^{2}}\right)}\left(\hat{B} \Pi_{p}^{\mathrm{OPE}}\right)}{\hat{B} \Pi_{p}^{\mathrm{OPE}}}$. 


\section{Numerical results and discussion}

This section is devoted to the numerical analysis of the sum rules for the mass and residue obtained in the previous section at nuclear matter. We discuss how the results in a dense medium deviate from those obtained via vacuum sum rules. For this aim, we need the numerical values of the quark masses as well as the in-medium quark-quark, quarkgluon and gluon-gluon condensates that are calculated in $[9,10,13,15]$. Each condensate at dense nuclear medium $\left(\langle\mathcal{O}\rangle_{\rho_{N}}\right)$ is written in terms of its vacuum values $\left(\langle\mathcal{O}\rangle_{0}\right)$ and its value between one-nucleon states $\left(\langle\mathcal{O}\rangle_{N}\right)$ at the low nuclear density limit, i.e. $\langle\mathcal{O}\rangle_{\rho_{N}}=\langle 0|\mathcal{O}| 0\rangle+\frac{\rho_{N}}{2 M_{N}}\langle N|\mathcal{O}| N\rangle=$ $\langle\mathcal{O}\rangle_{0}+\frac{\rho_{N}}{2 M_{N}}\langle\mathcal{O}\rangle_{N}$. We collect the numerical values of the input parameters in Table 1.

Looking at the sum rules for the physical quantities under consideration we see that they include three auxiliary parameters, namely, the continuum threshold $s_{0}$, the Borel mass parameter $M^{2}$, and the mixing parameter $\beta$, which should be fixed at this point. The standard criteria in QCD sum rules demand that the physical quantities show good stability with respect to these quantities at their working regions. As the mass sum rule is the ratio of two sum rules (see Eq. 33) including these auxiliary parameters, it may not lead to a reliable region. For this reason, we use the sum rule for the

Table 1 Numerical values for input parameters [9, 10, 13, 15]. The value presented for $\rho_{N}$ corresponds to the nuclear matter saturation density which is used in numerical analysis

\begin{tabular}{ll}
\hline Input parameters & Values \\
\hline$p_{0}$ & $1 \mathrm{GeV}$ \\
$m_{u}$ & $2.3 \mathrm{MeV}$ \\
$m_{d}$ & $4.8 \mathrm{MeV}$ \\
$\rho_{N}$ & $(0.11)^{3} \mathrm{GeV}^{3}$ \\
$\left\langle q^{\dagger} q\right\rangle_{\rho_{N}}$ & $\frac{3}{2} \rho_{N}$ \\
$\langle\bar{q} q\rangle_{0}$ & $(-0.241)^{3} \mathrm{GeV}^{3}$ \\
$m_{q}$ & $0.5\left(m_{u}+m_{d}\right)$ \\
$\sigma_{N}$ & $0.045 \mathrm{GeV}$ \\
$\langle\bar{q} q\rangle_{\rho_{N}}$ & $\langle\bar{q} q\rangle_{0}+\frac{\sigma_{N}}{2 m_{q}} \rho_{N}$ \\
$\left\langle q^{\dagger} g_{s} \sigma G q\right\rangle_{\rho_{N}}$ & $-0.33 \mathrm{GeV}^{2} \rho_{N}$ \\
$\left\langle q^{\dagger} i D_{0} q\right\rangle_{\rho_{N}}$ & $0.18 \mathrm{GeV}_{N}$ \\
$\left\langle\bar{q} i D_{0} q\right\rangle_{\rho_{N}}$ & $\frac{3}{2} m_{q} \rho_{N} \simeq 0$ \\
$m_{0}^{2}$ & $0.8 \mathrm{GeV}^{2}$ \\
$\left\langle\bar{q} g_{s} \sigma G q\right\rangle_{0}$ & $m_{0}^{2}\langle\bar{q} q\rangle_{0}$ \\
$\left\langle\bar{q} g_{s} \sigma G q\right\rangle_{\rho_{N}}$ & $\left\langle\bar{q} g_{s} \sigma G q\right\rangle_{0}+3 \mathrm{GeV}^{2} \rho_{N}$ \\
$\left\langle\bar{q} i D_{0} i D_{0} q\right\rangle_{\rho_{N}}$ & $0.3 \mathrm{GeV} \rho_{N}-\frac{1}{8}\left\langle\bar{q} g_{s} \sigma G q\right\rangle_{\rho_{N}}$ \\
$\left\langle q^{\dagger} i D_{0} i D_{0} q\right\rangle_{\rho_{N}}$ & $0.031 \mathrm{GeV}^{2} \rho_{N}-\frac{1}{12}\left\langle q^{\dagger} g_{s} \sigma G q\right\rangle_{\rho_{N}}$ \\
$\left\langle\frac{\alpha_{s}}{\pi} G^{2}\right\rangle_{0}$ & $(0.33 \pm 0.04)^{4} \mathrm{GeV}^{4}$ \\
$\left\langle\frac{\alpha_{s}}{\pi} G^{2}\right\rangle_{\rho_{N}}$ & $\left\langle\frac{\alpha_{s}}{\pi} G^{2}\right\rangle_{0}-0.65 \mathrm{GeV}_{N}$ \\
\hline &
\end{tabular}

residue to find the reliable regions for the auxiliary parameters. The working region for the Borel mass parameter is found as follows. The upper bound on this parameter is found by demanding that the contributions of the higher states and continuum are sufficiently suppressed and the ground state constitutes a large part of the whole dispersion integral, i.e.

$\frac{\int_{0}^{s_{0}} \rho(s) e^{-s / M^{2}}}{\int_{0}^{\infty} \rho(s) e^{-s / M^{2}}}>1 / 2$

should be satisfied. The lower bound on $M^{2}$ is calculated requiring that the perturbative part exceeds the nonperturbative one and the contributions of the operators with higher dimensions are small, i.e. the OPE converges. These requirements lead to the interval $0.8 \mathrm{GeV}^{2} \leqslant M^{2} \leqslant$ $1.2 \mathrm{GeV}^{2}$ for the Borel mass squared. The continuum threshold is not totally arbitrary, but it depends on the energy of the first excited state with the same quantum numbers as the interpolating current. We choose the interval $s_{0}=(1.5-$ 2.0) $\mathrm{GeV}^{2}$ for this parameter. Our numerical calculations show that, in this interval, the physical quantities depend weakly on this parameter and the results show good stability with respect to the variations of the Borel mass parameter in its working region.

Finally, the physical quantities under consideration should be independent of the mixing parameter $\beta$. To find the working region for this parameter, we look at the variation of the residue of the nucleon with respect to this parameter. To better cover the whole range of $-\infty \leqslant \beta \leqslant \infty$, which can mathematically be taken values in by this parameter, we plot the residue with respect to $x=\cos \theta$, where $\beta=\tan \theta$ at fixed values of the continuum threshold and Borel mass parameter for both nuclear medium and vacuum in Fig. 1. From this figure, we see that in the intervals $-1 \leqslant x \leqslant-0.5$ and $0.5 \leqslant x \leqslant 1$ the residues $\lambda_{N}^{*}$ and $\lambda_{N}$ are practically independent of this parameter. Moreover, the results of residues very weakly depend on the continuum threshold in these intervals. Note that the Ioffe current corresponding to $x \approx-0.71$ is included by these intervals. Here, we should mention that, as we said before, since the mass sum rule is the ratio of two sum rules, the unstable points of the two sum rules in nominator and denominator coincide and cancel each other. The masses in nuclear matter and vacuum show roughly good stabilities with respect to $x$ in the whole $-1 \leqslant x \leqslant 1$ region (see Fig. 2).

Having calculated the working regions, we discuss the variations of the masses and residues both in nuclear matter and vacuum with respect to the variations of the auxiliary parameters and look for the shifts in these parameters due to the nuclear medium by comparison of the results obtained in the nuclear matter as well as the vacuum. For this aim, in Figs. 3 and 4, we depict the variations of the residues and masses in the presence of nuclear matter and vacuum with respect to the Borel mass parameter at different fixed values of the 

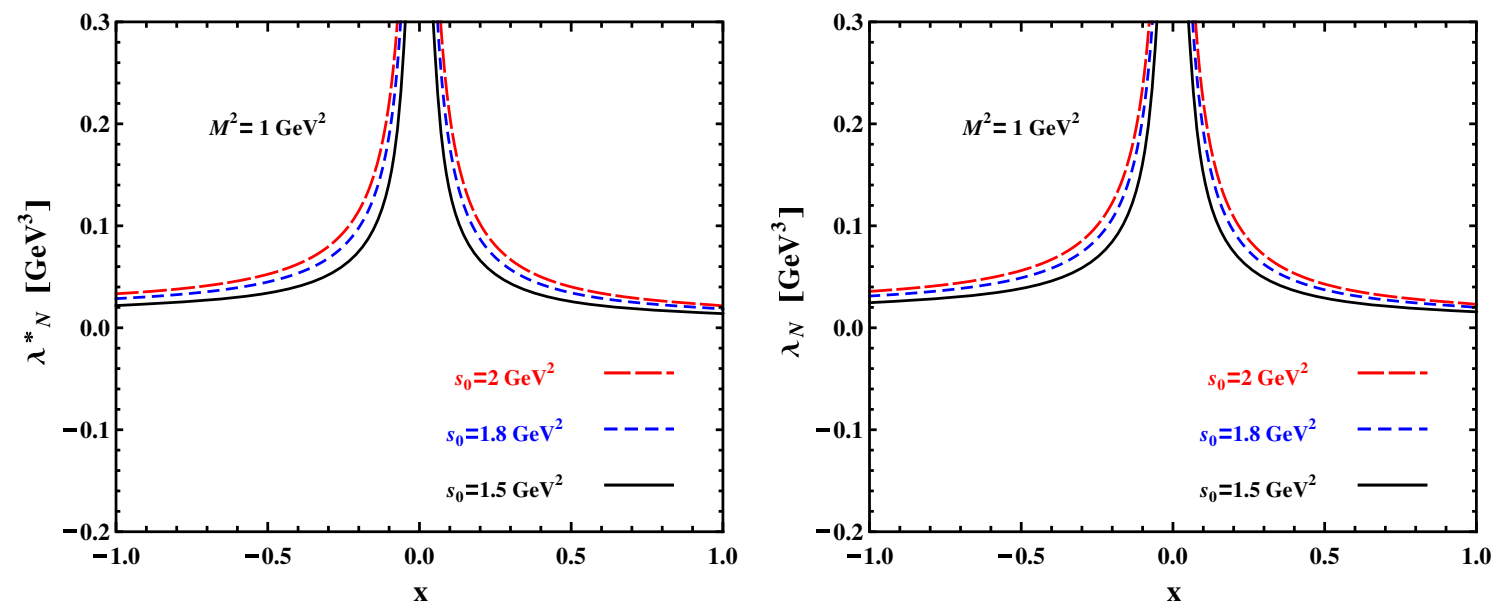

Fig. 1 The residue in nuclear matter versus $x$ (left panel). The residue in vacuum versus $x$ (right panel)
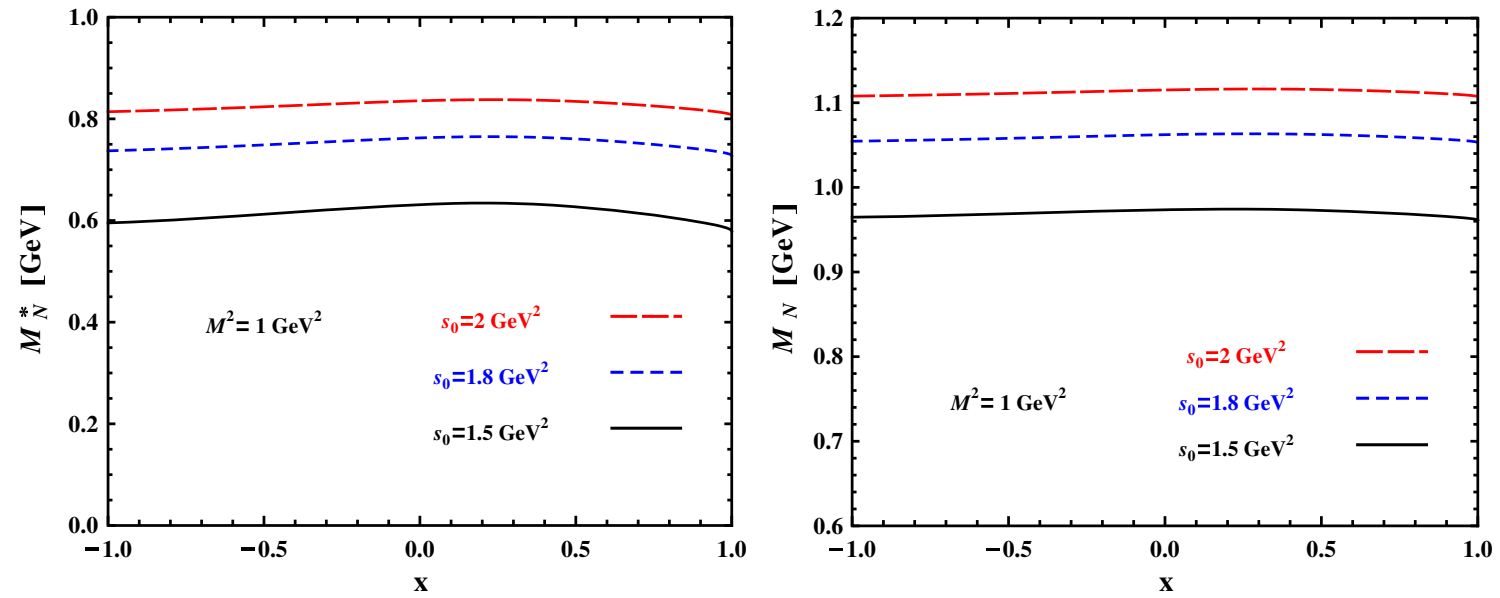

Fig. 2 The nucleon mass in nuclear matter versus $x$ (left panel). The nucleon mass in vacuum versus $x$ (right panel)
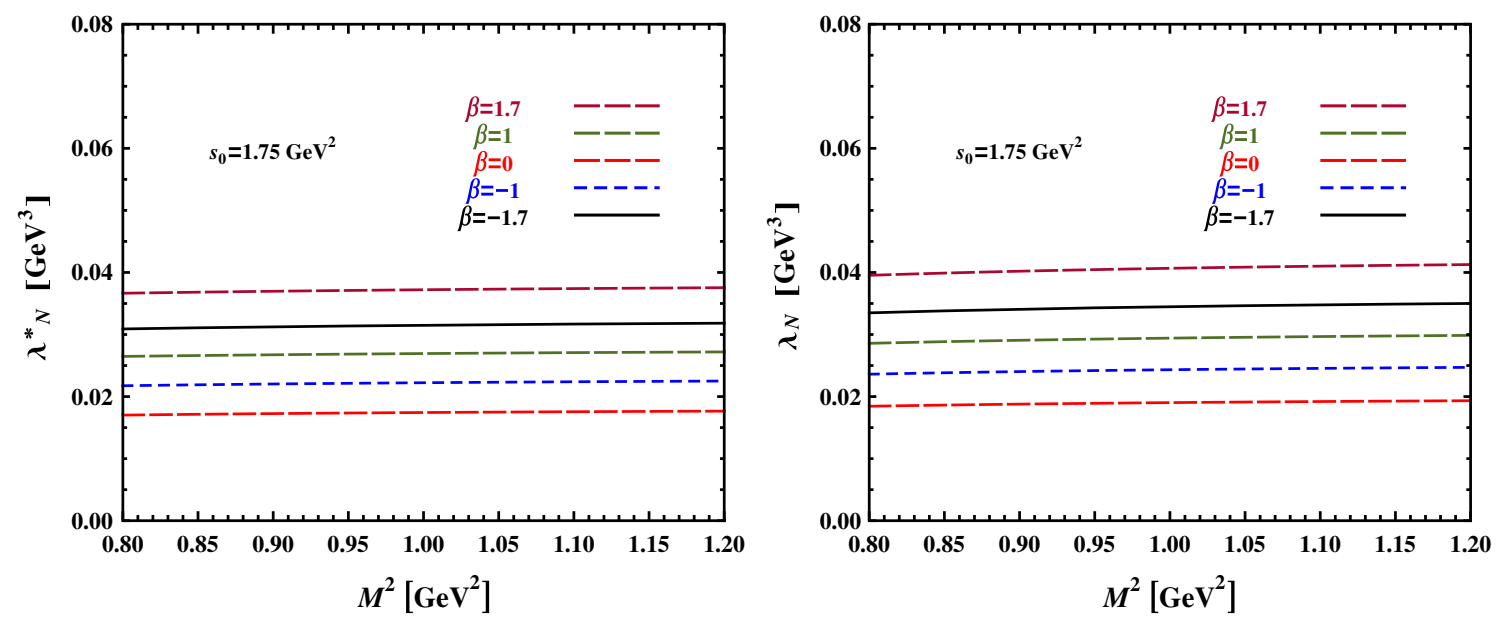

Fig. 3 The residue in nuclear matter versus Borel mass $M^{2}$ (left panel). The residue in vacuum versus Borel mass $M^{2}$ (right panel) 

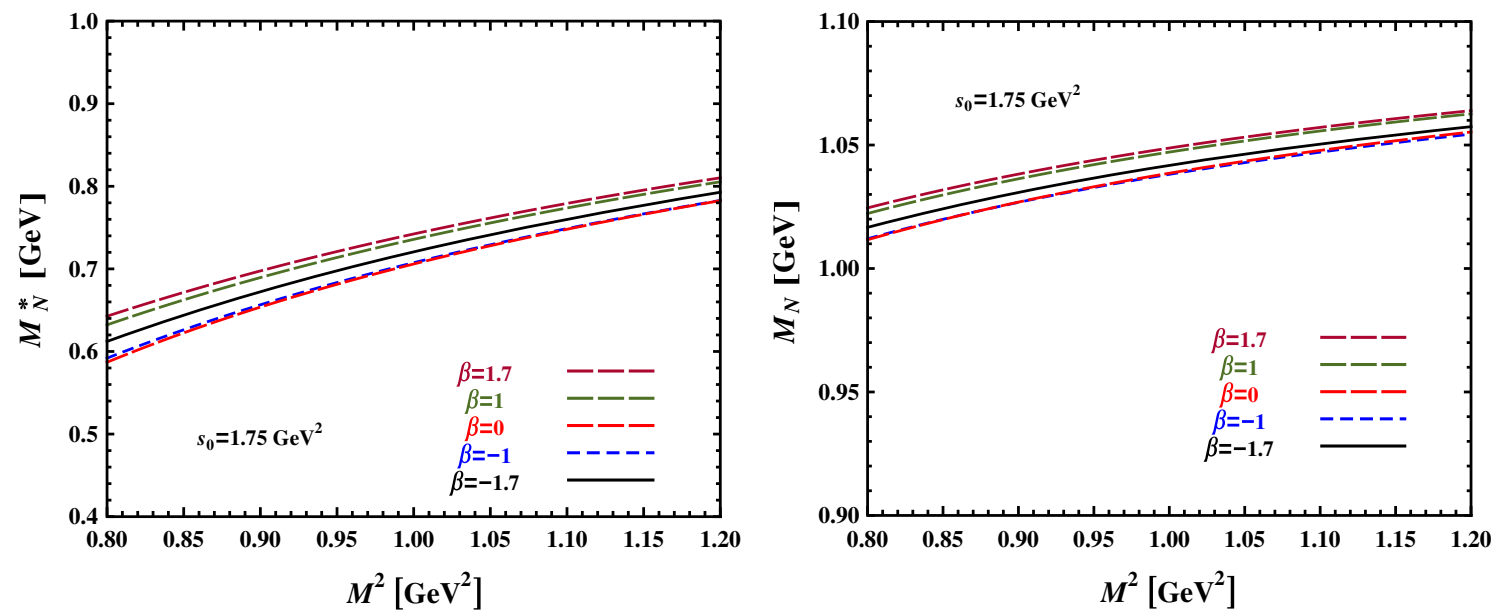

Fig. 4 The nucleon mass in nuclear matter versus Borel mass $M^{2}$ (left panel). The nucleon mass in vacuum versus Borel mass $M^{2}$ (right panel)

Table 2 Average values of the masses and residues squared in nuclear matter and vacuum obtained from a sum rules analysis and the comparison of the results with the existing results of the vacuum sum rules for the Ioffe current [42]; and the value of the mass obtained via the Ioffe current in vacuum considering the strangeness content of the nucleon [43]

\begin{tabular}{lllll}
\hline & $m_{N}^{*}(\mathrm{GeV})$ & $m_{N}(\mathrm{GeV})$ & $\lambda_{N}^{* 2}\left(\mathrm{GeV}^{6}\right)$ & $\lambda_{N}^{2}\left(\mathrm{GeV}^{6}\right)$ \\
\hline Present work & $0.723 \pm 0.122$ & $1.045 \pm 0.076$ & $0.0009 \pm 0.0004$ & $0.0011 \pm 0.0005$ \\
Ioffe & - & 0.985 & - & $0.0012 \pm 0.0006$ \\
nasrallah & - & $0.990 \pm 0.050$ & - & - \\
\hline
\end{tabular}

$\beta$ and $s_{0}$ picked from their working regions. These figures also indicate that the physical quantities under consideration vary weakly with respect to the auxiliary parameters in their working regions.

As obtained from Figs. 3 and 4, we depict the average values of the residues squared and masses of the nucleon both for the nuclear medium and vacuum in Table 2 and compare our results with the existing results obtained via the Ioffe current using the vacuum sum rules in this table. Note that in this table $m_{N}$ and $\lambda_{N}$ are the mass and residue of the nucleon in vacuum and these are, respectively, obtained from $m_{N}^{*}$ and $\lambda_{N}^{*}$ when $\rho_{N}=0$ is set. From this table, we conclude that the average values for the residue squared and mass when $\rho_{N} \rightarrow 0$ are consistent with the values obtained using the Ioffe current and vacuum sum rules $[42,43]$ within the errors. We also see that the average values of those quantities in nuclear matter show considerable shifts compared to the vacuum results. To see better how the results of the residue and mass in nuclear matter deviate from those of the vacuum, we depict the variations of the ratios of $\lambda_{N}^{*} / \lambda_{N}$ and $m_{N}^{*} / m_{N}$ as well as the percentages of the shifts with respect to the Borel mass squared in Figs. 5 and 6 at different fixed values of the parameter $\beta$ and the continuum threshold $s_{0}$. With a quick glance at these figures, we observe that the mass and residue of the nucleon show considerable shifts from their vacuum values and the shifts are negative. In the case of the residue, the shift grows roughly increasing the value of the Borel mass parameter. However, in the case of the mass, the shift deceases considerably when we increase the value of the Borel mass parameter in its working region. Our numerical results show that, on average, the values of the residue and mass decrease by about 15 and $32 \%$, respectively, compared to their values in vacuum. Note that we have used the nuclear matter saturation density, $\rho_{N}^{\text {sat }}=(0.11)^{3} \mathrm{GeV}^{3}$, in our numerical analysis as well as the density dependence of some condensates in leading order (see Table 1). To check whether the results depend linearly on the nuclear matter density or not, we depict the dependences of, for instance, $m_{N}^{*} / m_{N}$ and $\lambda_{N}^{*} / \lambda_{N}$ on $\rho_{N} / \rho_{N}^{\text {sat }}$ in Fig. 7 for fixed values of $s_{0}, M^{2}$ and $\beta$. From this figure we see that $\lambda_{N}^{*} / \lambda_{N}$ is exactly linear and $m_{N}^{*} / m_{N}$ is roughly linear in terms of $\rho_{N} / \rho_{N}^{\text {sat }}$ and they considerably decrease increasing the value of the nuclear matter density.

At the end of this section, we would like to extract the values of the vector and scalar self-energies of the nucleon in nuclear matter. From our analysis we obtain the values $\Sigma_{S}=$ $-(322 \pm 51) \mathrm{MeV}$ and $\Sigma_{0}=(420 \pm 65) \mathrm{MeV}$ for the scalar and time-like vector self-energies of the nucleon in nuclear medium, respectively. When we compare these results with the ones obtained using a model independent study in [32, 33], i.e., $\Sigma_{S}=-(400-450) \mathrm{MeV}$ and $\Sigma_{0}=(350-400) \mathrm{MeV}$, we see that our result on time-like vector self-energies of the 

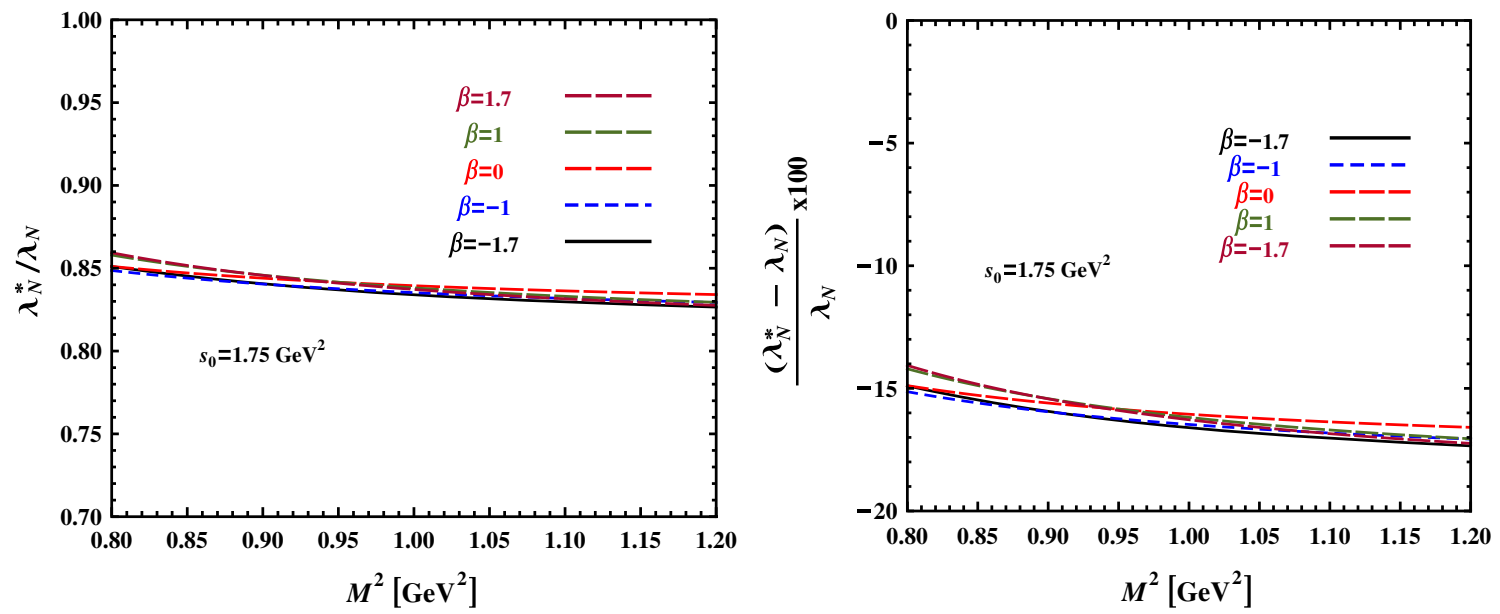

Fig. $5 \lambda_{N}^{*} / \lambda_{N}$ versus Borel mass parameter $M^{2}$ (left panel). The percentage of the shift in the residue of the nucleon in nuclear matter compared to its vacuum value (right panel)
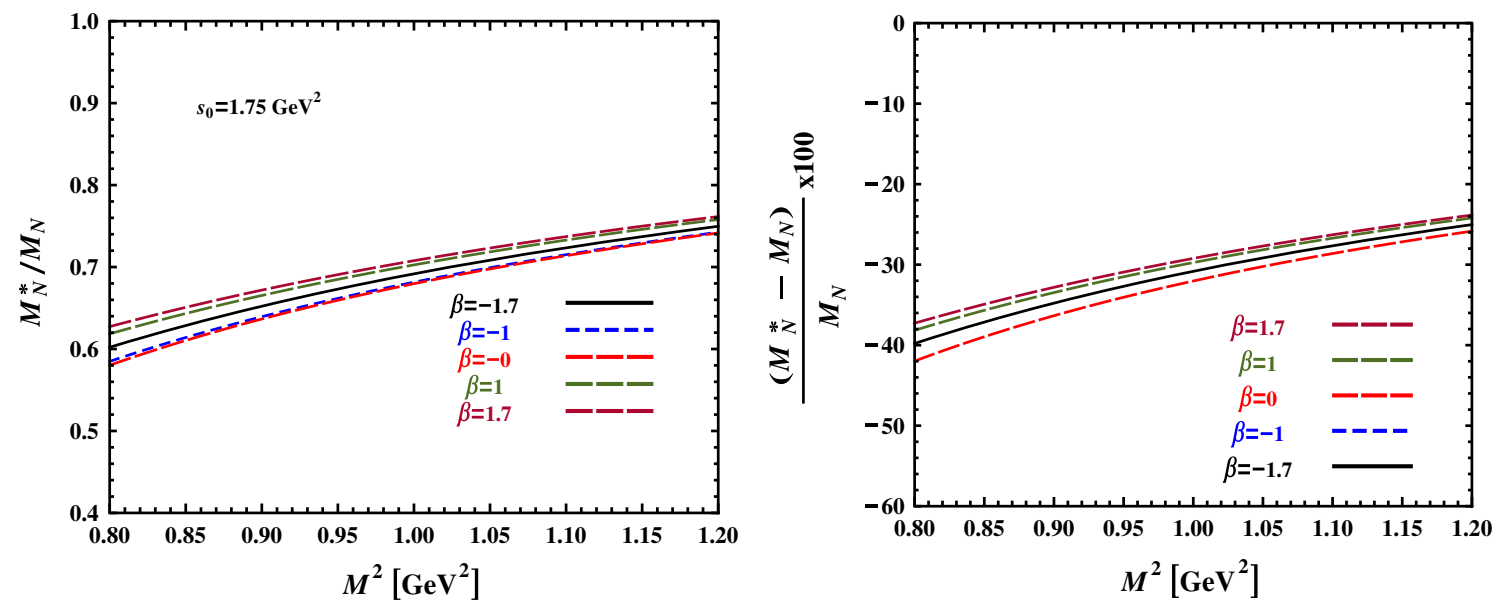

Fig. $6 m_{N}^{*} / m_{N}$ versus Borel mass parameter $M^{2}$ (left panel). The percentage of the shift in the mass of the nucleon in nuclear matter compared to its vacuum value (right panel)
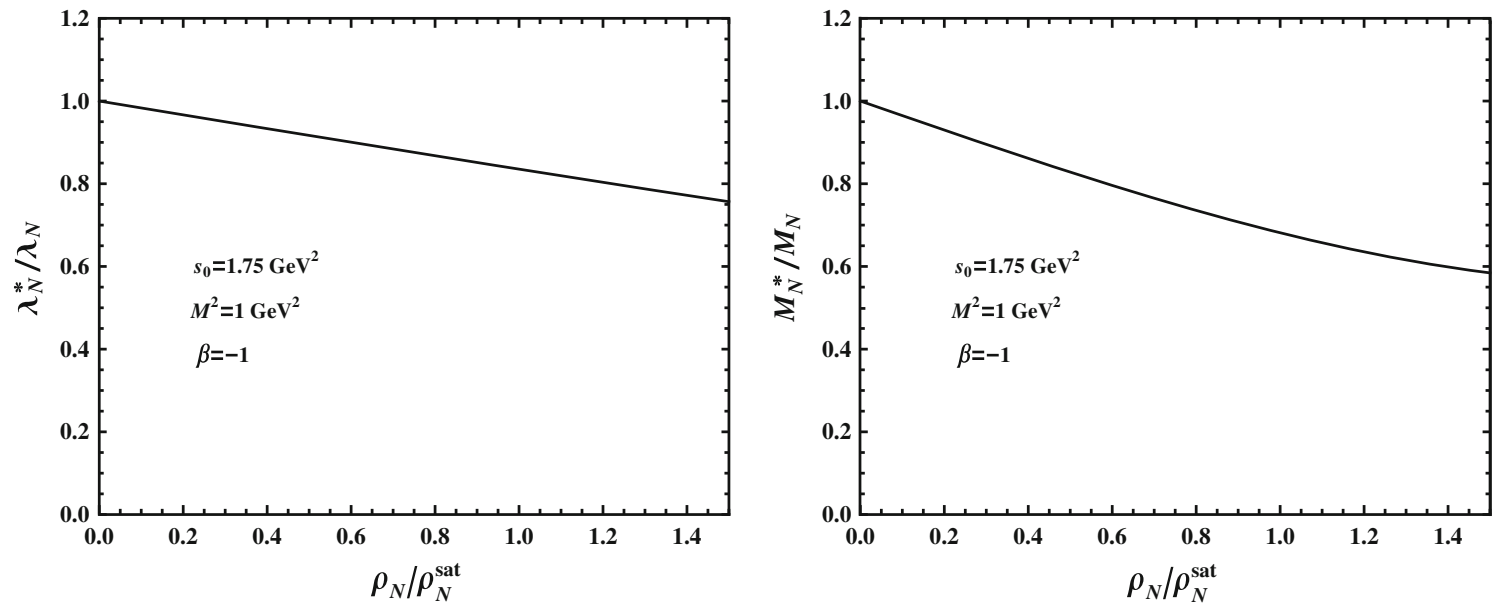

Fig. $7 \lambda_{N}^{*} / \lambda_{N}$ versus $\rho_{N} / \rho_{N}^{\text {sat }}\left(\right.$ left panel). $m_{N}^{*} / m_{N}$ versus $\rho_{N} / \rho_{N}^{\text {sat }}$ (right panel) 
nucleon is consistent with the predictions of $[32,33]$ within the errors. In the case of the scalar self-energy, although our result is consistent with those of $[32,33]$ in sign, its absolute value is smaller than those of $[32,33]$.

\section{Conclusion}

In the present work, we studied some properties of the nucleon in the nuclear matter using the QCD sum rules. In particular, we calculated the mass and residue of the nucleon in nuclear medium and looked for the shifts of the results compared to their vacuum values. Using the interpolating current of the nucleon with an arbitrary mixing parameter, we extended the previous works on the mass of the nucleon discussed, which mainly use the Ioffe current. We also extended the recent study [30] on the residue of the nucleon pole, which uses a special current corresponding to an axial-vector diquark coupled to a quark, by introducing the arbitrary mixing parameter into the interpolating current. We found the working regions for the three main auxiliary parameters entering the sum rules using the obtained QCD sum rule for the residue. Using the working regions obtained for the continuum threshold, the Borel mass parameter, and the mixing parameter $\beta$ entering the interpolating current, we depicted the variations of the physical quantities under consideration with respect to the variations of the auxiliary parameters. We observed considerable negative shifts in the values of the mass and residue of the nucleon in nuclear matter compared to their values in vacuum. The results of the residue and mass reduce by about 15 and $32 \%$, respectively, due to the nuclear medium. We also extracted the values of the scalar and time-like vector self-energies of the nucleon in the nuclear medium and compared the results obtained with the predictions of model-independent studies [32,33].

The obtained results for the mass and residue in nuclear matter can be used in theoretical determinations of the electromagnetic properties of the nucleon and its strong couplings to other hadrons in the nuclear medium.

Open Access This article is distributed under the terms of the Creative Commons Attribution License which permits any use, distribution, and reproduction in any medium, provided the original author(s) and the source are credited.

Funded by $\mathrm{SCOAP}^{3}$ / License Version CC BY 4.0.

\section{References}

1. W. Erni et al., PANDA Collaboration. arXiv:0903.3905 [hep-ex]

2. B. Friman et al., The CBM Physics Book: Compressed Baryonic Matter in Laboratory Experiments (Springer, Heidelberg, 2011)
3. E.G. Drukarev, E.M. Levin, Pisma Zh. Eksp. Teor. Fiz. 48, 307 (1988)

4. E.G. Drukarev, E.M. Levin, Nucl. Phys. A 511, 679 (1990)

5. E.G. Drukarev, E.M. Levin, Nucl. Phys. A 516, 715(E) (1990)

6. T. Hatsuda, H. Hogaasen, M. Prakash, Phys. Rev. Lett. 66, 2851 (1991)

7. C. Adami, G.E. Brown, Z. Phys. A 340, 93 (1991)

8. R.J. Furnstahl, D.K. Griegel, T.D. Cohen, Phys. Rev. C 46, 1507 (1992)

9. X. Jin, T.D. Cohen, R.J. Furnstahl, D.K. Griegel, Phys. Rev. C 47, $2882(1993)$

10. X. Jin, M. Nielsen, T.D. Cohen, R.J. Furnstahl, D.K. Griegel, Phys. Rev. C 49, 464 (1994)

11. T.D. Cohen, R.J. Furnstahl, D.K. Greigel, Phys. Rev. Lett. 67, 961 (1991)

12. H. Hogaasen, Acta Phys. Pol. B 22, 1123 (1991)

13. T.D. Cohen, R.J. Furnstahl, D.K. Griegel, Phys. Rev. C 45, 1881 (1992)

14. E.G. Drukarev, Prog. Part. Nucl. Phys. 50, 659 (2003)

15. T.D. Cohen, R.J. Furnstahl, D.K. Griegel, X. Jin, Prog. Part. Nucl. Phys. 35, 221 (1995)

16. Z.-G. Wang, T. Huang, Phys. Rev. C 84, 048201 (2011)

17. Z.-G. Wang, Phys. Rev. C 85, 045204 (2012)

18. Z.-G. Wang, Eur. Phys. J. C 71, 1816 (2011)

19. T. Hatsuda, S.H. Lee, Phys. Rev. C 46, R34 (1992)

20. M. Asakawa, C.M. Ko, Nucl. Phys. A 560, 399 (1993)

21. M. Asakawa, C.M. Ko, Phys. Rev. C 48, R526 (1993)

22. X. Jin, D.B. Leinweber, Phys. Rev. C 52, 3344 (1995)

23. F. Klingl, N. Kaiser, W. Weise, Nucl. Phys. A 624, 527 (1997)

24. S. Leupold, U. Mosel, Phys. Rev. C 58, 2939 (1998)

25. A. Hayashigaki, Phys. Lett. B 487, 96 (2000)

26. E.G. Drukarev, M.G. Ryskin, V.A. Sadovnikova, T. Gutsche, A. Faessler, Phys. Rev. C 69, 065210 (2004)

27. T. Hilger, R. Thomas, B. Kämpfer, Phys. Rev. C 79, 025202 (2009)

28. Y. Yasui, K. Sudoh, Phys. Rev. C 87, 015202 (2013)

29. R. Thomas, T. Hilger, B. Kampfer, Nucl. Phys. A 795, 19 (2007)

30. S. Mallik, S. Sarkar, Eur. Phys. J. C 65, 247 (2010)

31. E.G. Drukarev, M.G. Ryskin, V.A. Sadovnikova, arXiv:1312.1449 [hep-ph]

32. O. Plohl, C. Fuchs, E.N.E. van Dalen, Phys. Rev. C 73, 014003 (2006)

33. O. Plohl, C. Fuchs, A. Faessler, in Proceedings of the XLIV International Winter Meeting on Nuclear Physics, Bormio, Italy, arXiv:Nucl-th/0603070

34. T.M. Aliev, K. Azizi, A. Ozpineci, M. Savci, Phys. Rev. D 77, 114014 (2008)

35. T.M. Aliev, A. Ozpineci, M. Savci, V.S. Zamiralov, Phys. Rev. D 80, 016010 (2009)

36. T.M. Aliev, K. Azizi, M. Savci, Nucl. Phys. A 847, 101 (2010)

37. D.B. Leinweber, Phys. Rev. D 51, 6383 (1995)

38. E. Stein, P. Gornicki, L. Mankiewicz, A. Schafer, W. Greiner, Phys. Lett. B 343, 369 (1995)

39. M.A. Shifman, A.I. Vainshtein, V.I. Zakharov, Nucl. Phys. B 147, 385 (1979)

40. P. Colangelo, A. Khodjamirian, in At the Frontier of Particle Physics/Handbook of QCD, vol. 3, ed. by M. Shifman (World Scientific, Singapore, 2001), p. 1495

41. L.J. Reinders, H. Rubinstein, S. Yazaki, Phys. Rep. 127, 1 (1985)

42. B.L. Ioffe, Nucl. Phys. B 188, 317 (1981)

43. N.F. Nasrallah, K. Schilcher, Phys. Rev. C 89, 045202 (2014) 